\title{
6 Stärken und Limitationen der Arbeit
}

Die besondere Stärke des vorliegenden Literaturreviews stellt die systematische Vorgehensweise dar. Zwei Personen (DE und SE) führten unabhängig voneinander die umfassende Literaturrecherche durch. Auch die Bewertung der methodologischen Qualität der eingeschlossenen Studien wurde von zwei Personen unabhängig voneinander durchgeführt und bei Unstimmigkeiten die Stärken und Schwächen der einzelnen Studien diskutiert bis ein Konsens erreicht werden konnte. Diese Vorgehensweise erhöht die Objektivität der vorliegenden Arbeit.

Als Limitation muss an dieser Stelle angeführt werden, dass die Literaturrecherche ausschließlich auf deutschsprachige und englischsprachige Publikationen beschränkt war. Eine Schwäche der eingeschlossenen Studien ist, dass diese ausschließlich Beobachtungsstudien sind. Damit können zwar Zusammenhänge aufgezeigt werden, jedoch keine Kausalitäten bzw. können keine Rückschlüsse auf Therapieoptionen gezogen werden. Eine Beschränkung auf Beobachtungsstudien wurde deshalb durchgeführt, da diese besonders geeignet sind Zusammenhänge aufzuzeigen. Eine Einbeziehung von Studien aller Studiendesigns hätte den Rahmen der vorliegenden Masterabeit überschritten.

Da die AutorInnen der einzelnen Studien viele unterschiedliche Definitionen bzw. Instrumente zur Erkennung der Sarkopenie eingesetzt haben, können die Ergebnisse nur begrenzt gegenübergestellt und verglichen werden. Auch können aus diesem Hintergrund heraus, wie bereits erwähnt, keine allgemeingültigen Aussagen getroffen werden. Für zukünftige Projekte ist es deshalb vernünftig anzudenken, nur solche Studien einzubeziehen, die ähnliche Definitionen und Instrumente zur Erkennung der Sarkopenie zu Grunde legen.

(C) Springer Fachmedien Wiesbaden 2016

D. Eglseer, Sarkopenie und Ernährungszustand,

Best of Pflege, DOI 10.1007/978-3-658-15160-7_6 